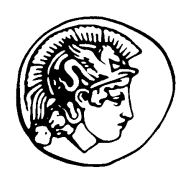

PERGAMON International Journal of Non-Linear Mechanics 36 (2001) 1075-1084

\title{
Some physical models with Minkowski spacetime structure and Lorentz group symmetry
}

\author{
Hong-Ki Hong ${ }^{\mathrm{a}}$, Chein-Shan Liu ${ }^{\mathrm{b}, *}$ \\ ${ }^{a}$ Department of Civil Engineering, Taiwan University, Taipei 106-17, Taiwan \\ ${ }^{\mathrm{b}}$ Department of Mechanical and Marine Engineering, National Taiwan Ocean University, Keelung 20224, Taiwan
}

Received 10 November 1999; accepted 12 June 2000

\begin{abstract}
Projecting the linear equation $\dot{\mathbf{X}}=\mathbf{A X}, \mathbf{A} \in \operatorname{so}(n, 1)$, yields a primitive model. It is a prototype of several physical models, namely perfect elastoplasticity (on phase), spacetime of special relativity, special relativistic mechanics and so on, all of which may endow a Minkowskian spacetime and the Lorentz group left acts on it. The mathematical structure of the perfect elastoplastic equations is then compared with those of the spacetime of special relativity and of the special relativistic equations of motion of a massive charged particle; their similarities in group properties and subtle differences in phase spaces are discussed. It is remarkable that the evolutions from elastic constitutive equations to elastoplastic constitutive equations and from the Newtonian equations to the special relativistic equations are very similar in several facets, notably (a) from a linear theory to a non-linear theory, (b) the state space being enlarged from the usual Euclidean space to Minkowski spacetime, (c) from an $n$-space to a cone of $(n+1)$-space, (d) from a non-bounded state space to a bounded state space, (e) from a non-causal relation of states to a causal relation of augmented states. (C) 2001 Elsevier Science Ltd. All rights reserved.
\end{abstract}

Keywords: Minkowski spacetime; Lorentz group; Perfect elastoplasticity; Special relativity; Special relativistic mechanics

\section{Introduction}

The perfect elastoplastic model with or without considering large deformation has been transformed into a two-phase linear system with an on-off switch, and has been examined further to explore its Minkowski spacetime structure and Lorentz group symmetry in [1,2]. Once we have learned the Lorentz group properties of plasticity, our curiosity is naturally drawn to comparing plas-

\footnotetext{
*Corresponding author. Tel.: +886-2-2462-2192; fax: + 886-2-2462-0836.

E-mail address: csliu@earth.me.ntou.edu.tw (C.-S. Liu).
}

ticity and relativity, which might bear certain similarities. It is known that scientists have considerable experience that further progress can be clarified and simplified by introducing the concepts of Minkowski spacetime and Lorentz group to treat the equations of special relativity; see, for example [3-6]. Scientists have also gained very deep insight into electromagnetic field theory through the skill in the notion of four vectors of spacetime and energy-momentum. Therefore, cross references among the theories will enhance our understanding of their intrinsic subtleties, as will be pursued in Section 4.

Although our consideration is concentrated on perfect elastoplastic models, the issue is considered 
quintessential in the modelling of plasticity in general, since as we shall realize later the underlying structure of these simple models must be the core and foundation on which more complicated models can be based and from which a hierarchy of constitutive models can be built up. We hope the current paper would shed light on, and bring new momentum to, the study of plasticity on one hand, and, on the other hand, it might introduce this fascinating area to mathematicians who want to know more sciences in which they can cultivate their mathematics than classical mechanics, quantum mechanics, electrodynamics, cosmology, fluid mechanics and elasticity.

To avoid misunderstanding we add that the present paper addresses one of the constitutive model issues rather than the (initial-)boundary value problems of stress analysis (e.g. [7]), and that the paper digs out the hidden Lorentz group symmetry in the constitutive model, which happens to be mathematically analogous somewhat to the relativistic effect, rather than analyzes a mechanical problem of such high-speed or high strain rate that the relativistic effect has to be taken into account.

\section{Perfect elastoplasticity}

\subsection{Constitutive postulates}

The model of perfect elastoplasticity may be postulated as follows (e.g. $[1,8,9])$ :

$$
\begin{aligned}
& \dot{\mathbf{q}}=\dot{\mathbf{q}}^{\mathrm{e}}+\dot{\mathbf{q}}^{\mathrm{p}}, \\
& \dot{\mathbf{Q}}=k_{e} \dot{\mathbf{q}}^{\mathrm{e}}, \\
& \mathbf{Q} \dot{q}_{0}=Q^{0} \dot{\mathbf{q}}^{\mathrm{p}}, \\
& \|\mathbf{Q}\| \leqslant Q^{0}, \\
& \dot{q}_{0} \geqslant 0, \\
& \|\mathbf{Q}\| \dot{q}_{0}=Q^{0} \dot{q}_{0} .
\end{aligned}
$$

As usual, $\|\mathbf{Q}\|:=\sqrt{\mathbf{Q}^{t} \mathbf{Q}}$ denotes the Euclidean length of the vector $\mathbf{Q}$, while the superscript $t$ de- notes the transpose. Here $\mathbf{Q}$ and $\dot{\mathbf{q}}$ are a pair of dual vectors in $n$-dimensional Euclidean space $\mathbb{E}^{n}$; $\mathbf{Q}=\operatorname{col}\left(Q^{1}, Q^{2}, \ldots, Q^{n}\right)=\operatorname{col}\left(Q_{1}, Q_{2}, \ldots, Q_{n}\right) \quad$ denotes the generalized stress vector and $\dot{\mathbf{q}}=$ $\operatorname{col}\left(\dot{q}_{1}, \dot{q}_{2}, \ldots, \dot{q}_{n}\right)=\operatorname{col}\left(\dot{q}^{1}, \dot{q}^{2}, \ldots, \dot{q}^{n}\right)$ denotes the generalized strain rate vector. The above constitutive model is re-postulated from the celebrated Prandtl-Reuss equation formulated by Prandtl [10] and Reuss [11].

\subsection{Two-phase system with an on-off switch}

Substituting Eqs. (2) and (3) into Eq. (1) gives

$\dot{\mathbf{Q}}+\frac{\dot{q}_{0}}{q_{y}} \mathbf{Q}=k_{e} \dot{\mathbf{q}}$,

where $q_{y}:=Q^{0} / k_{e}$ is known as the generalized yield strain. The term $\dot{q}_{0}$ is subjected to the following on-off switching criteria for the mechanism of plasticity:

$\dot{q}_{0}= \begin{cases}\frac{1}{Q^{\circ}} \mathbf{Q}^{\mathrm{t}} \dot{\mathbf{q}}>0 & \text { if }\|\mathbf{Q}\|=Q^{0} \text { and } \mathbf{Q}^{\mathrm{t}} \dot{\mathbf{q}}>0, \\ 0 & \text { if }\|\mathbf{Q}\|<Q^{0} \text { or } \mathbf{Q}^{\mathrm{t}} \dot{\mathbf{q}} \leqslant 0 .\end{cases}$

Based on the criteria and the complementary trios (4)-(6), there are precisely two phases: the on-phase in which $\dot{q}_{0}>0$ and $\|\mathbf{Q}\|=Q^{0}$ and the off-phase in which $\dot{q}_{0}=0$ and $\|\mathbf{Q}\| \leqslant Q^{0}$; see e.g. [1]. The complementary trios (4)-(6) further specify the admissible region in the phase plane $\left(\dot{q}_{0},\|\mathbf{Q}\| / Q^{0}\right)$ as shown in Fig. $1 b$, on which the admissible regions are shaded. We set $\dot{q}_{0}=0$ in Eq. (7) for elasticity, whose admissible region in the phase plane $\left(\dot{q}_{0},\|\mathbf{Q}\| / Q^{0}\right)$ is shown in Fig. 1a.

Using Eq. (8) to eliminate $\dot{q}_{0}$ from Eq. (7) results in a two-phase non-linear system:

$\dot{\mathbf{Q}}= \begin{cases}k_{e} \dot{\mathbf{q}}-k_{e} \frac{\mathbf{Q}^{\mathrm{t} \dot{\mathbf{q}}}}{\left(Q^{0}\right)^{2}} \mathbf{Q} & \text { if }\|\mathbf{Q}\|=Q^{0} \text { and } \mathbf{Q}^{\mathrm{t}} \dot{\mathbf{q}}>0, \\ k_{e} \dot{\mathbf{q}} & \text { if }\|\mathbf{Q}\|<Q^{0} \text { or } \mathbf{Q}^{\mathrm{t}} \dot{\mathbf{q}} \leqslant 0,\end{cases}$

of which the latter is linear and represents an instantaneous response, and the former is a system of non-linear differential equations. This is a nonlinear representation in the $n$-dimensional space of $\mathbf{Q}=\left(Q^{1}, Q^{2}, \ldots, Q^{n}\right)$. 

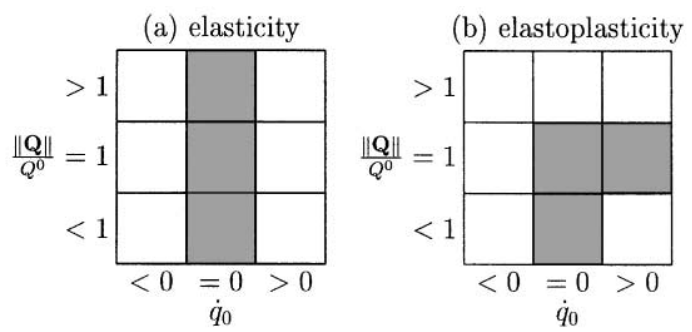

(c) comparison model

(d) primitive model

(e) sp. rel. spacetime
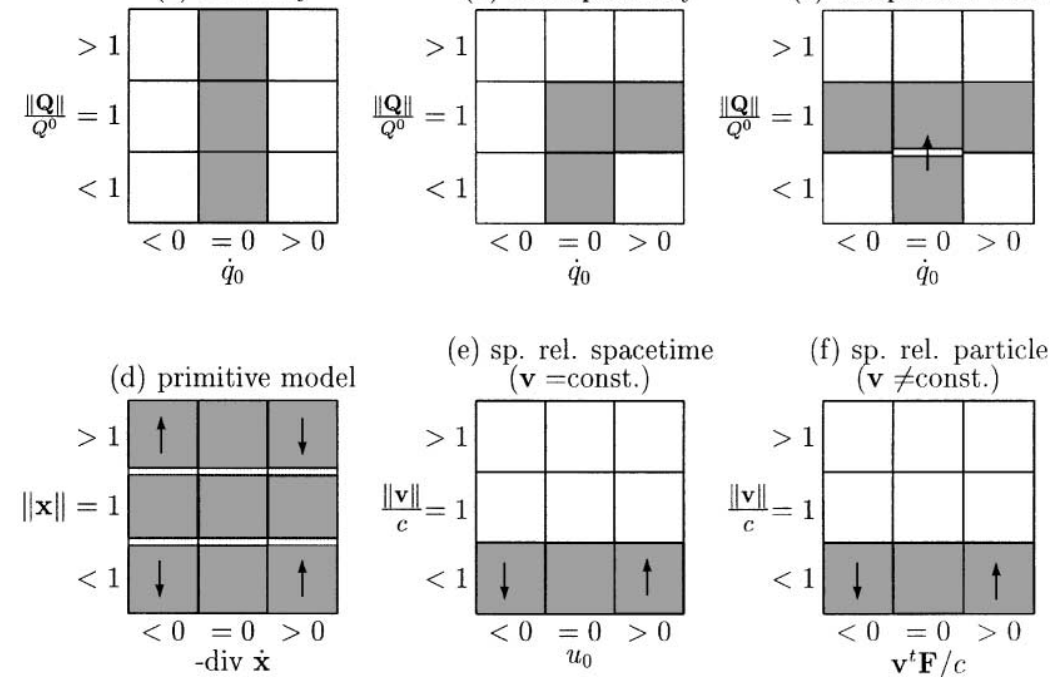

(f) sp. rel. particle

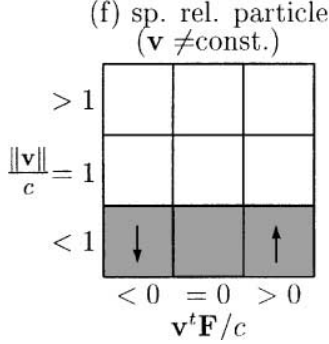

Fig. 1. Comparison of the six models on the phase planes, on which the admissible regions are shaded and a white strip between boxes represents no trespassing, but an arrow over a white strip permits one-way trespassing, whereas an arrow in a box indicates the ordinate is strictly monotonic in that box. Even the last five models all possess the Lorentz group symmetry structures, their phase planes are so different.

\subsection{Minkowski spacetime}

Let us define

$\mathbf{X}=\left[\begin{array}{l}\mathbf{X}^{\mathbf{s}} \\ X^{0}\end{array}\right]=\left[\begin{array}{l}X^{1} \\ X^{2} \\ \vdots \\ X^{n} \\ X^{0}\end{array}\right]:=\frac{\exp \left(q_{0} / q_{y}\right)}{Q^{0}}\left[\begin{array}{l}\mathbf{Q} \\ Q^{0}\end{array}\right]$

$=\frac{\exp \left(q_{0} / q_{y}\right)}{Q^{0}}\left[\begin{array}{l}Q^{1} \\ Q^{2} \\ \vdots \\ Q^{n} \\ Q^{0}\end{array}\right]$

and call it the $(n+1)$-dimensional augmented stress vector. Recently, Hong and Liu [1] have recast model (1)-(6) postulated in the generalized stress space of $\mathbf{Q}$ into a model in the augmented stress space of $\mathbf{X}$ :

$\left[\begin{array}{cc}\mathbf{I}_{n} & \mathbf{0}_{n \times 1} \\ \mathbf{0}_{1 \times n} & \mathbf{X}^{\mathbf{t}} \mathbf{g X}\end{array}\right] \dot{\mathbf{X}}=\frac{1}{q_{y}}\left[\begin{array}{cc}\mathbf{0}_{n \times n} & \dot{\mathbf{q}} \\ \mathbf{0}_{1 \times n} & 0\end{array}\right] \mathbf{X}$,
$\mathbf{X}^{\mathrm{t}} \mathbf{g X} \leqslant 0$,

$\dot{X}^{0}=X^{0} \frac{\mathbf{Q}^{\mathbf{t}}}{Q^{0}} \frac{\dot{\mathbf{q}}}{q_{y}} \geqslant 0$,

in terms of the Minkowski metric (in the space-like convention)

$\mathbf{g}=\left[\begin{array}{ll}\mathbf{g}_{s s} & \mathbf{g}_{s 0} \\ \mathbf{g}_{0 s} & \mathbf{g}_{00}\end{array}\right]=\left[\begin{array}{cc}\mathbf{I}_{n} & \mathbf{0}_{n \times 1} \\ \mathbf{0}_{1 \times n} & -1\end{array}\right]$,

where $\mathbf{I}_{n}$ is the identity tensor of order $n$. The vector space of augmented stresses $\mathbf{X}$ endowed with the Minkowski metric tensor $\mathbf{g}$ is referred to as Minkowski spacetime and designated as $\mathbb{M}^{n+1}$.

Spacetime of this sort underlying the constitutive theory may be called internal spacetime, because we can think of it as having to do with the intrinsic nature of the mechanical behavior of the solid materials or the structural members which the constitutive model describes, rather than their position or motion in ordinary (external) space and (external) time. Thus the "temporal" coordinate $X^{0}$ and the "spatial" coordinates $\mathbf{X}^{\mathrm{s}}=\left(X^{1}, X^{2}, \ldots, X^{n}\right)^{\mathrm{t}}$ may be thought of as the internal time and the internal space, respectively. 
Moreover, we have the augmented stress (linear differential) equation

$\dot{\mathbf{X}}=\mathbf{A X}$

with the control tensor

$$
\begin{aligned}
& \mathbf{A}:= \\
& \left\{\begin{array}{l}
\frac{1}{q_{v}}\left[\begin{array}{cc}
\mathbf{0}_{n \times n} & \dot{\mathbf{q}} \\
\dot{\mathbf{q}}^{\mathrm{t}} & 0
\end{array}\right] \text { if } \mathbf{X}^{\mathrm{t}} \mathbf{g X}=0 \text { and } \frac{\mathrm{d}}{\mathrm{d} t}\left[\left(\mathbf{X}^{\mathrm{s}}\right)^{\mathrm{t}} \mathbf{g}_{s s} \mathbf{X}^{\mathrm{s}}\right]>0, \\
\frac{1}{q_{v}}\left[\begin{array}{cc}
\mathbf{0}_{n \times n} & \dot{\mathbf{q}} \\
\mathbf{0}_{1 \times n} & 0
\end{array}\right] \text { if } \mathbf{X}^{\mathrm{t}} \mathbf{g X}<0 \text { or } \frac{\mathrm{d}}{\mathrm{d} t}\left[\left(\mathbf{X}^{\mathrm{s}}\right)^{\mathrm{t}} \mathbf{g}_{s s} \mathbf{X}^{\mathrm{s}}\right] \leqslant 0 .
\end{array}\right.
\end{aligned}
$$

It is remarkable that in the augmented stress space the two-phase system becomes linear. All together it is a two-phase linear system with an on-off switch. The last row of the off-phase's $\mathbf{A}$ is full of zeros since $X^{0}$ is constant (i.e. $q_{0}$ is fixed) in the off phase. Thus, we have revealed the linearity of the perfect elastoplasticity model both in the on and off phases.

\subsection{The Lorentz group}

Denote by $I_{\text {on }}$ an open, maximal, continuous time interval during which the mechanism of plasticity is on exclusively. The solution of Eq. (15) with Eq. $(16)_{1}$ can be expressed in the following augmented stress transition formula:

$\mathbf{X}(t)=\left[\mathbf{G}(t) \mathbf{G}^{-1}\left(t_{1}\right)\right] \mathbf{X}\left(t_{1}\right), \quad \forall t, t_{1} \in I_{\mathrm{on}}$,

in which $\mathbf{G}(t)$, known as the fundamental solution of Eq. (15), is a transformation tensor satisfying

$$
\begin{aligned}
\dot{\mathbf{G}}(t) & =\mathbf{A}(t) \mathbf{G}(t), \\
\mathbf{G}(0) & =\mathbf{I}_{n+1},
\end{aligned}
$$

where the control tensor $\mathbf{A}$ in the on-phase satisfies

$\mathbf{A}^{\mathrm{t}} \mathbf{g}+\mathbf{g A}=\mathbf{0}$.

Recall that the complete homogeneous Lorentz group $O(n, 1)$ is the group of all invertible linear transformations in Minkowski spacetime which leave the Minkowski metric invariant, and that the proper orthochronous Lorentz group $\mathrm{SO}_{o}(n, 1)$ is a subgroup of $O(n, 1)$ in which the transformations are proper (i.e. orientation preserving, namely the determinants of the transformations being +1 ) and orthochronous (i.e. time-orientation preserving, namely the 00th entry of the matrix representations of the transformations being positive); see, for example, Cornwell [12].

A straightforward generalization of the Lorentz group $\operatorname{SO}_{o}(n, 1)$ is a Poincare group, which is a semi-direct product of a translation group with the Lorentz group. In this way we can take the (linear or non-linear) kinematic hardening effect into consideration [13]. In the on-phase the augmented stress vectors remain on the cone. A generalization for this is to replace the cone by a hyperboloid, resulting in a model capable of accounting for the isotropic hardening (or softening). A simultaneous generalization of the two thus renders mixed hardening.

\subsection{Boost transformation}

Consider a rectilinear generalized strain path with non-zero constant rate

$\dot{\mathbf{q}}=$ constant $\neq \mathbf{0}$.

The constitutive response can be determined exactly, $[1,9]$, and it may be recast in the form of Eq. (17) with a boost transformation:

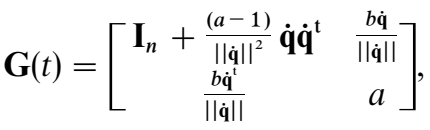

where

$a:=\cosh \left(t\|\dot{\mathbf{q}}\| / q_{y}\right), \quad b:=\sinh \left(t\|\dot{\mathbf{q}}\| / q_{y}\right)$.

(Cf. with Eq. (37) in Section 4.2.) It is not difficult to see that the above $\mathbf{G}$ satisfies

$\mathbf{G}^{\mathrm{t}} \mathbf{g G}=\mathbf{g}$,

$\operatorname{det} \mathbf{G}=1$,

$G_{0}^{0}>0$,

where $\mathbf{g}$ is a Minkowskian metric given in Eq. (14). Therefore, the curve $\mathbf{G}(t)$ going through the identity $\mathbf{G}(0)=\mathbf{I}_{n+1}$ at $t=0$ is an one-parameter subgroup of the Lorentz group $\mathrm{SO}_{o}(n, 1)$. 


\section{Abstract dynamic system - a primitive model}

Zeeman [14] was able to show that the causality assumption in Minkowski spacetime renders a composition of a translation, a dilation, and a proper orthochronous Lorentz transformation. To begin with we will focus on the most essential among the three - the proper orthochronous Lorentz group $\mathrm{SO}_{o}(n, 1)$, which gives uniquely the real Lie algebra $\operatorname{so}(n, 1)$ (to within isomorphism).

By Eq. (20), A is an element of the real Lie algebra $\operatorname{so}(n, 1)$ of the Lorentz group $\mathrm{SO}_{o}(n, 1)$. An element $\mathbf{A}$ of the real Lie algebra $\operatorname{so}(n, 1)$ which satisfies Eq. (20) has the general form

$\mathbf{A}=\left[\begin{array}{ll}\boldsymbol{\Omega} & \mathbf{u} \\ \mathbf{u}^{\mathbf{t}} & 0\end{array}\right]$,

where $\quad \mathbf{u}=\left(u_{1}, u_{2}, \ldots, u_{n}\right)^{\mathrm{t}}:=\left(A_{10}, A_{20}, \ldots, A_{n 0}\right)^{\mathrm{t}}$ and $\boldsymbol{\Omega}$ is skew-symmetric, i.e. $\boldsymbol{\Omega}=-\boldsymbol{\Omega}^{\mathrm{t}}$, in which the entries $\Omega_{i j}:=A_{i j}$ for $1 \leqslant i<j \leqslant n$. Note that the earlier form of $\mathbf{A}$ as given in Eq. (16) has zero $\boldsymbol{\Omega}$ and is, therefore, less general than it may. We may generalize the perfect elastoplastic model by including a non-vanishing, skew-symmetric tensor $\boldsymbol{\Omega}$ if we no longer assume negligibly small spinning but instead consider large deformation and rotation [2].

We choose to assume $X^{0}>0$ and to define the state $n$-vector

$\mathbf{x}=\left(x_{1}, x_{2}, \ldots, x_{n}\right)^{\mathrm{t}}:=\left(X^{1}, X^{2}, \ldots, X^{n}\right)^{\mathrm{t}} / X^{0}$.

Indeed it is a projection. Utilizing this and Eq. (26), we can partition Eq. (15) into

$\frac{\mathrm{d}}{\mathrm{d} t}\left[\begin{array}{c}X^{0} \mathbf{x} \\ X^{0}\end{array}\right]=\left[\begin{array}{cc}\mathbf{\Omega} & \mathbf{u} \\ \mathbf{u}^{\mathrm{t}} & 0\end{array}\right]\left[\begin{array}{c}X^{0} \mathbf{x} \\ X^{0}\end{array}\right]$.

Consequently, the augmented state equation (15) becomes the state equation

$\dot{\mathbf{x}}=\mathbf{\Omega} \mathbf{x}-\left(\mathbf{x}^{\mathrm{t}} \mathbf{u}\right) \mathbf{x}+\mathbf{u}$

after eliminating $X^{0}$. We can identify three vector fields in the non-linear differential equation: one spinning $\boldsymbol{\Omega x}$, one dissipative $\left(\mathbf{x}^{\mathbf{t}} \mathbf{u}\right) \mathbf{x}$, and one direct $\mathbf{u}$.

For later references we may call the dynamic system derived so far - mainly represented by the non-linear state equation (29) — as the primitive model [15]. To summarize, the principle of causal- ity in Minkowski spacetime $\mathbb{M}^{n+1}$ implies the Lorentz group $\mathrm{SO}_{o}(n, 1) \ni \mathbf{G}$, which gives uniquely the real Lie algebra $\operatorname{so}(n, 1) \ni \mathbf{A}$, which in turn gives the flow $\mathbf{X}(t)$. Finally, projecting the flow equation $\dot{\mathbf{X}}=\mathbf{A X}$ yields the primitive model. Note that the primitive model is not the perfect elastoplastic model, but the perfect elastoplastic model in the on-phase behaves like the primitive model.

Take the divergence of Eq. (29) with respect to $\mathbf{x}$, giving

$-\operatorname{div} \dot{\mathbf{x}}=(n+1) \mathbf{x}^{\mathrm{t}} \mathbf{u}$.

The inner product of Eq. (29) with $\mathbf{x}$ leads to

$\frac{\mathrm{d}\|\mathbf{x}\|^{2}}{\mathrm{~d} t}=2 \mathbf{x}^{\mathrm{t}} \mathbf{u}\left(1-\|\mathbf{x}\|^{2}\right)$.

A set $\mathscr{S}$ in $\mathbb{R}^{n}$ is said to be an invariant set of Eq. (29) if, for any point $\mathbf{p} \in \mathscr{S}$ the solution curve through p belongs to $\mathscr{S}$ for $t$ in $(-\infty, \infty)$; see, e.g. [16]. In view of Eq. (31) it is obvious that $\mathscr{S}:=\{\mathbf{x} \mid\|\mathbf{x}\|=1\}$ is an invariant set of Eq. (29). In case the initial condition is $\left\|\mathbf{x}\left(t_{i}\right)\right\|=1$, it remains $\|\mathbf{x}(t)\|=1$ for all $t>t_{i}$; however, in case the initial condition is $\left\|\mathbf{x}\left(t_{i}\right)\right\|<1$, it is always $\|\mathbf{x}(t)\|<1$ for all $t>t_{i}$, and in case the initial condition is $\left\|\mathbf{x}\left(t_{i}\right)\right\|>1$, it is always $\|\mathbf{x}(t)\|>1$ for all $t>t_{i}$. Thus, the three states $\|\mathbf{x}(t)\|<1,\|\mathbf{x}(t)\|=1$ and $\|\mathbf{x}(t)\|>1$ are disconnected as shown in Fig. 1d. The state $\|\mathbf{x}(t)\|=1$ is the $\omega$-limit set for arbitrary initial conditions under the condition $\mathbf{x}^{\mathrm{t}} \mathbf{u}>0$, and the $\alpha$-limit set for arbitrary initial conditions under the condition $\mathbf{x}^{\mathbf{t}} \mathbf{u}<0$.

\section{Comparison of the models}

In this section we compare the perfect elastoplastic model with the so-called comparison model, the spacetime of special relativity, and the equations of special relativistic particle mechanics, for they happen to bear some sorts of internal symmetry related to the Lorentz group.

\subsection{Comparison model}

The concept of comparison model (a model of fictitious material or member) has been used by some authors, e.g. Hill [17] and Sewell [18], to 
derive a sufficient condition for the uniqueness of boundary value problem for an associated flow model. Indeed, the comparison model may be modified from Eqs. (1)-(6) by lifting the constraint on $\dot{q}_{0}$ in Eq. (5), namely

$$
\begin{aligned}
& \dot{\mathbf{q}}=\dot{\mathbf{q}}^{\mathrm{e}}+\dot{\mathbf{q}}^{\mathrm{p}}, \\
& \dot{\mathbf{Q}}=k_{e} \dot{\mathbf{q}}^{\mathrm{e}}, \\
& \mathbf{Q} \dot{q}_{0}=Q^{0} \dot{\mathbf{q}}^{\mathrm{p}}, \\
& \|\mathbf{Q}\| \leqslant Q^{0}, \\
& \|\mathbf{Q}\| \dot{q}_{0}=Q^{0} \dot{q}_{0} .
\end{aligned}
$$

Consequently, except for the switching criteria and the causality relation, all the other relevant results derived earlier are also valid for the comparison model, for which $\|\mathbf{Q}\|=Q^{0}$ for all $t$ after initial yielding. Hence, for this model we have the admissible regions in the phase plane $\left(\dot{q}_{0},\|\mathbf{Q}\| / Q^{0}\right)$ as shown in Fig. 1c. For comparison purposes the facts and results of elastoplasticity, comparison model, and elasticity are summarized in Table 1 and Figs. 1a-c.

\subsection{Comparison with special relativity}

Having learned the Lorentz group symmetry of plasticity, we are eager to compare it with the structure of relativity. Since our interest is on their similarities and distinctions, the following presenta- tion about relativity theory itself (in this subsection and the subsection which follows) will be kept to minimum where the notation and equations suffice to make comparison.

Let $c$ denote the speed of light in vacuo. An event is a point

$\mathbf{X}=\left(X^{1}, X^{2}, X^{3}, X^{0}\right)^{\mathrm{t}}:=(x, y, z, c t)^{\mathrm{t}}$

in four-dimensional Minkowski spacetime $\mathbb{M}^{1+3}$, which is endowed with the Minkowski metric (in the time-like convention)

$\mathbf{g}:=\operatorname{diag}(-1,-1,-1,1)$

and in which the square of the Minkowskian length of a differential element of a world line (or a string of events)

$(\mathrm{d} X)^{2}:=\mathrm{d} \mathbf{X}^{\mathrm{t}} \mathbf{g} \mathrm{d} \mathbf{X}>0$

is invariant with respect to different inertial observing frames. The same event is described by frames $S(\alpha)$ and $S(0)$ as $\mathbf{X}(\alpha)$ and $\mathbf{X}(0)$, respectively. If $S(0)$ moves with a constant velocity $\mathbf{v}$ relative to $S(\alpha)$, in which the rapidity $\alpha$ is related to the relative velocity $\mathbf{v}$ by

$\cosh \alpha:=\gamma:=\frac{1}{\sqrt{1-v^{2} / c^{2}}}, \quad v:=\|\mathbf{v}\|$,

then the special theory of relativity asserts that

\begin{tabular}{|c|c|c|c|c|}
\hline \multirow{2}{*}{$\begin{array}{l}\text { Abstract } \\
\text { dynamic system }\end{array}$} & \multirow[t]{2}{*}{ Elasticity } & \multicolumn{2}{|c|}{ Perfect elastoplasticity } & \multirow[t]{2}{*}{ Comparison model } \\
\hline & & Off & On & \\
\hline $\begin{array}{l}\text { Augmented } \\
\text { state space }\end{array}$ & $\mathbb{E}^{n} \times\left\{X^{0}\left(t_{i}\right)\right\}$ & $\mathbb{D}^{n} \times\left\{X^{0}(t) \mid t \geqslant t_{i}\right\}$ & $\left\{\mathbb{M}^{n+1} \mid\right.$ cone $\}$ & $\mathbb{D}^{n} \times\left\{X^{0}\left(t_{i}\right)\right\} \rightarrow\left\{\mathbb{M}^{n+1} \mid\right.$ cone $\}$ \\
\hline Cone & No & Interior & Truncated $\left(X^{0} \geqslant 1\right)$ & Positive $\left(X^{0}>0\right)$ \\
\hline $\mathbf{X}$ & $X^{0}=X^{0}\left(t_{i}\right)$ & $\mathbf{X}^{\mathrm{t}} \mathbf{g X} \leqslant 0$ & $\mathbf{X}^{\mathrm{t}} \mathbf{g} \mathbf{X}=0$ & $\mathbf{X}^{\mathrm{t}} \mathbf{g X} \leqslant 0$ \\
\hline$\dot{\mathbf{X}}$ & $\dot{\mathbf{X}}^{t} \mathbf{g} \dot{\mathbf{X}}>0$ & $\dot{\mathbf{X}}^{\dagger} \mathbf{g} \dot{\mathbf{X}}>0$ & $\dot{\mathbf{X}}^{\dagger} \mathbf{g} \dot{\mathbf{X}} \geqslant 0$ & $\dot{\mathbf{X}}^{\dagger} \mathbf{g} \dot{\mathbf{X}} \geqslant 0$ \\
\hline Control matrix & A (off) & A (off) & A (on) & $\mathbf{A}($ off $) \rightarrow \mathbf{A}($ on $)$ \\
\hline Causality & No & No & Yes & Lost \\
\hline$\dot{X}^{0}$ & $\dot{X}^{0}=0$ & $\dot{X}^{0}=0$ & $\dot{X}^{0}>0$ & $\dot{X}^{0}$ no constraint \\
\hline$u_{0}$ & $\dot{q}_{0}=0$ & $\dot{q}_{0}=0$ & $\dot{q}_{0}>0$ & $\dot{q}_{0}$ no constraint \\
\hline On-off switch & No & Can switch on & Can switch off & Can on/never off \\
\hline State space & No constraint & $\|\mathbf{Q}\| \leqslant Q^{0}$ & $\|\mathbf{Q}\|=Q^{0}$ & $\|\mathbf{Q}\| \leqslant Q^{0}$ \\
\hline Group & $\operatorname{SE}(n)$ & $\mathrm{SE}(n) \rightleftarrows \mathrm{PSO}_{o}(n, 1)$ & & $\mathrm{SE}(n) \rightarrow \mathrm{PSO}_{o}(n, 1)$ \\
\hline
\end{tabular}

$\mathbf{X}(\alpha)=\mathbf{G}(\alpha) \mathbf{X}(0)$,

Table 1

Comparisons of elastoplasticity, comparison model, and elasticity 
where

$$
\mathbf{G}:=\left[\begin{array}{cc}
\mathbf{I}_{3}+\frac{\gamma-1}{v^{2}} \mathbf{v} \mathbf{v}^{\mathbf{t}} & \gamma \frac{\mathbf{v}}{c} \\
\gamma / \frac{\mathbf{v}^{\mathbf{v}}}{c} & \gamma
\end{array}\right]
$$

is called the boost transformation (see, for example, [19]).

Consider an infinite number of inertial frames $S(\alpha), \alpha \geqslant 0$, where no privilege is implied in the frame labelled with $\alpha=0$ or with any other number. Then similar to Eqs. (15) and (17)-(19), we get from Eq. (36) the event observation (linear differential) equation

$\frac{\mathrm{d} \mathbf{X}}{\mathrm{d} \alpha}=\mathbf{A X}$

with the control matrix

$$
\mathbf{A}:=\left[\begin{array}{cc}
\mathbf{0}_{3 \times 3} & \frac{\mathbf{v}}{v} \\
\frac{\mathbf{v}^{\mathrm{v}}}{v} & 0
\end{array}\right] .
$$

From the above two equations one easily deduces the following:

$u_{0}:=\frac{\mathrm{d} X^{0} / \mathrm{d} \alpha}{X^{0}}=\frac{X^{0}(0) \sinh \alpha+\left(\mathbf{v}^{\mathrm{t}} / v\right) \mathbf{X}^{\mathrm{s}}(0) \cosh \alpha}{X^{0}(0) \cosh \alpha+\left(\mathbf{v}^{\mathrm{t}} / v\right) \mathbf{X}^{\mathrm{s}}(0) \sinh \alpha}$,

where $\mathbf{X}^{\mathrm{s}}:=\left(X^{1}, X^{2}, X^{3}\right)^{\mathrm{t}}$ is the spatial part of $\mathbf{X}$.

We note that the event observation transformation formula (36), the boost transformation (37), the event observation equation (38), and the (constant) control matrix (39) of special relativity are math- ematically analogous to, respectively, the augmented state transition formula (17), the augmented state fundamental matrix (21), the augmented state equation (15), and the (constant) control matrix (16) ${ }_{1}$ of perfect elastoplasticity under rectilinear generalized strain paths, with the following similarities:

$\mathbf{v} \simeq \dot{\mathbf{q}}, \quad v \simeq\|\dot{\mathbf{q}}\|, \quad \gamma=\cosh \alpha \simeq a$,

$\gamma \frac{v}{c}=\sinh \alpha \simeq b, \quad \alpha \simeq \frac{t\|\dot{\mathbf{q}}\|}{q_{y}}, \quad u_{0} \simeq \frac{\dot{q}_{0}}{q_{y}}$.

See also Fig. 1 and Table 2 for comparisons. Notice in particular that the constancy of the relative velocity ( $\mathbf{v}=$ constant $)$ between observers is correspondent to the constancy of the generalized strain rate $(\dot{\mathbf{q}}=$ constant). It is easy to see that the boost transformation $\mathbf{G}$ satisfies Eqs. (23)-(25); therefore, as well known, the $\mathbf{G}$ is an element of the proper orthochronous Lorentz group $\mathrm{SO}_{o}(1,3)$ acting on Minkowski spacetime $\mathbb{M}^{1+3}$.

\subsection{Comparison with special relativistic particle mechanics}

Within the realm of special relativity, the momentum $\mathbf{p}=\left(p^{1}, p^{2}, p^{3}\right)^{\mathrm{t}}$ and energy $E$ of a massive particle can be expressed, respectively, as

$\mathbf{p}=m \mathbf{v}$,
$E=m c^{2}$,

Table 2

\begin{tabular}{|c|c|c|c|}
\hline $\begin{array}{l}\text { Abstract } \\
\text { dynamic system }\end{array}$ & $\begin{array}{l}\text { Spacetime of } \\
\text { special relativity }\end{array}$ & $\begin{array}{l}\text { Special relativistic } \\
\text { mechanics }\end{array}$ & $\begin{array}{l}\text { Perfect } \\
\text { elastoplasticity (on) }\end{array}$ \\
\hline Aug. state space & $\left\{\mathbf{X} \in \mathbb{M}^{1+3} \mid \mathbf{X}^{\mathbf{t}} \mathbf{g X}<0\right\}$ & $\left\{\mathbf{X} \in \mathbb{M}^{3+1} \mid \mathbf{X}^{\mathbf{t}} \mathbf{g X}<0\right\}$ & $\left\{\mathbf{X} \in \mathbb{M}^{n+1} \mid \mathbf{X}^{\mathbf{t}} \mathbf{g X}=0\right\}$ \\
\hline Cone & Full $\left(X^{0} \in \mathbb{R}\right)$ & Truncated $\left(X^{0} \geqslant c\right)$ & Truncated $\left(X^{0} \geqslant 1\right)$ \\
\hline Metric & $\operatorname{diag}(-1,-1,-1,1)$ & $\operatorname{diag}(1,1,1,-1)$ & block diag $\left(\mathbf{I}_{n},-1\right)$ \\
\hline Aug. state path & Time like & Space like & Space like \\
\hline Group & $\mathrm{SO}_{o}(1,3)$ & $\mathrm{SO}_{o}(3,1)$ & $\mathrm{SO}_{o}(n, 1)$ \\
\hline Asym. part of $\mathbf{A}$ & None & Due to magnetism & Due to spin \\
\hline State space & $\|\mathbf{v}\|<c$ & $\|\mathbf{v}\|<c$ & $\|\mathbf{Q}\|=Q^{0}$ \\
\hline$X^{0}$ & $c t \in \mathbb{R}$ & $c \gamma \geqslant c$ & $X^{0} \geqslant 1$ \\
\hline$u_{0}$ & $u_{0} \in \mathbb{R}$ & $\mathbf{v}^{t} \mathbf{F} / c \in \mathbb{R}$ & $\dot{q}_{0}>0$ \\
\hline$\|\mathbf{x}\| u_{0}=u_{0}$ & No & No & Yes \\
\hline On-off switch & No & No & Yes \\
\hline If $u_{0}=0$ & Galilean spacetime & Newtonian mechanics & Linear elasticity \\
\hline
\end{tabular}

Comparisons of spacetime of special relativity, special relativistic mechanics and perfect elastoplasticity 
where $m$ and $\mathbf{v}=\left(v^{1}, v^{2}, v^{3}\right)^{\mathrm{t}}$ are the relativistic mass and velocity (which is not necessarily constant), respectively, of the particle under investigation. It is postulated that $m$ depends on the particle speed $v:=\|\mathbf{v}\|, m>0,0 \leqslant v<c$, and $c$ is constant. The force $\mathbf{F}=\left(F^{1}, F^{2}, F^{3}\right)^{\mathrm{t}}$ is defined as the rate of change of the momentum $\mathbf{p}$, and the power $\mathbf{F}^{\mathbf{t}} \mathbf{v}$ is the rate of change of the energy $E$, namely

$\mathbf{F}=\frac{\mathrm{d}}{\mathrm{d} t} \mathbf{p}$,

$\mathbf{F}^{\mathrm{t}} \mathbf{v}=\frac{\mathrm{d}}{\mathrm{d} t} E$

In these respects the concepts of momentums, forces, and powers of Einstein and Newton are markedly the same.

Substituting Eqs. (42) and (43) into Eqs. (44) and (45), respectively, and then combining them in two different ways, we obtain two equations:

$m \dot{\mathbf{v}}=\mathbf{F}-\frac{\mathbf{F}^{\mathrm{t}} \mathbf{v}}{c^{2}} \mathbf{v}$,

$m v \dot{v}=\dot{m}\left(c^{2}-v^{2}\right)$,

the latter of which can be further integrated to the following famous relationship:

$m(v)=\frac{m_{0}}{\sqrt{1-v^{2} / c^{2}}}=\gamma m_{0}$,

in which the integration constant $m_{0}:=\left.m(v)\right|_{v=0}$ is the mass at rest, so called the rest mass.

In general the relativistic equations of motion with a given force are more difficult to solve than the corresponding non-relativistic equations with the same force because the non-linear terms $m_{0} \dot{\mathbf{v}} / \sqrt{1-\|\mathbf{v}\|^{2} / c^{2}}$ and $\left(\mathbf{F}^{\mathrm{t}} \mathbf{v}\right) \mathbf{v} / c^{2}$ appear in Eq. (46). It seems more convenient to define the proper time $\tau$ as follows:

$\mathrm{d} \tau=\sqrt{1-\frac{v^{2}}{c^{2}}} \mathrm{~d} t, \quad \tau=\tau_{0}+\int_{t_{0}}^{\mathrm{t}} \sqrt{1-\frac{v^{2}\left(t^{\prime}\right)}{c^{2}}} \mathrm{~d} t^{\prime}$,

where $\tau_{0}$ is the initial proper time and $t_{0}$ is the initial time. Because $0 \leqslant v<c$, the proper time is connected with the external time $t$ strictly monotonically. In terms of the proper time through Eqs.
(48) and (49), Eq. (46) can be rearranged to

$\frac{\mathrm{d} \mathbf{v}}{\mathrm{d} \tau}=\frac{1}{m_{0}} \mathbf{F}-\frac{\mathbf{F}^{\mathrm{t}} \mathbf{v}}{m_{0} c^{2}} \mathbf{v}$.

Comparing the two differential equations (50) and (9) 1 yields the following similarities:

$\mathbf{v} \simeq \mathbf{Q}, \quad \mathbf{F} \simeq \dot{\mathbf{q}}, \quad c \simeq Q^{0}, \quad \frac{1}{m_{0}} \simeq k_{e}, \quad \tau \simeq t$.

Both $c$ and $Q^{0}$ play the roles of bounded values in the corresponding equations, and $1 / m_{0}$ and $k_{e}$ are moduli. As noted in the last paragraph of Section 3, this type equations, Eqs. (50), (9) ${ }_{1}$ and (29), have an invariant set. However, for the special relativistic particle equations, the invariant set $\|\mathbf{v}\|=c$ is not reachable; conversely, for the perfect elastoplastic equations, the invariant set $\|\mathbf{Q}\|=Q^{0}$ is reachable through a different rule of elasticity, Eq. $(9)_{2}$. Upon reaching $\|\mathbf{Q}\|=Q^{0}$, Eq. (9) ${ }_{1}$ never allows $\mathbf{Q}$ return back to the set $\left\{\mathbf{Q} \mid\|\mathbf{Q}\|<Q^{0}\right\}$, so a concomitant switching rule as shown in Eq. (8) is designed such that the material can return to its elastic state.

Introduce the so-called four-velocity

$\mathbf{X}=\left(X^{1}, X^{2}, X^{3}, X^{0}\right)^{\mathrm{t}}:=\left(\gamma \mathbf{v}^{\mathrm{t}}, \gamma c\right)^{\mathrm{t}}=\left(\mathbf{p}^{\mathrm{t}}, E / c\right)^{\mathrm{t}} / m_{0}$,

the last equality of which expresses that the fourvector of momentum-energy (or simply the fourmomentum) $\left(\mathbf{p}^{\mathbf{t}}, E / c\right)^{\mathbf{t}}$ and the four-velocity $\mathbf{X}$ are indeed equivalent up to a proportionality constant $m_{0}$, the rest mass. By using Eqs. (44), (45), and (48)-(52), it is not difficult to prove the four-velocity (linear differential) equation

$$
\frac{\mathrm{d} \mathbf{X}}{\mathrm{d} \tau}=\mathbf{A X}
$$

with the control matrix

$$
\mathbf{A}:=\frac{1}{m_{0} c}\left[\begin{array}{llll}
0 & 0 & 0 & F^{1} \\
0 & 0 & 0 & F^{2} \\
0 & 0 & 0 & F^{3} \\
F^{1} & F^{2} & F^{3} & 0
\end{array}\right] .
$$
into

The restriction $v=\|\mathbf{v}\|<c$ may be translated

$\mathbf{X}^{\mathbf{t}} \mathbf{g X}=-c^{2}<0$, 
which indicates that the four-velocity $\mathbf{X}$ (or equivalently the four-vector of momentum-energy) of the particle must be located in the interior of the cone $\left\{\mathbf{X} \mid \mathbf{X}^{\mathbf{t}} \mathbf{g X}=0\right\}$ of the flat four-vector space of $\left(X^{1}, X^{2}, X^{3}, X^{0}\right)$ which is endowed with the Minkowski metric (in the space-like convention)

$\mathbf{g}:=\operatorname{diag}(1,1,1,-1)$

and in which the square of the Minkowskian length of a differential element of a space-like path is

$(\mathrm{d} X)^{2}:=\mathrm{d} \mathbf{X}^{\mathrm{t}} \mathbf{g} \mathrm{d} \mathbf{X}>0$.

Up to this point one may be amazed at the similarity between the governing equations of special relativistic mechanics and perfect elastoplasticity in the on-phase. Comparing the two differential equations (53) and (15) yields the following similarities:

$\mathbf{v} \simeq \mathbf{Q}, \quad \mathbf{F} \simeq \dot{\mathbf{q}}, \quad c \simeq Q^{0}$,

$\frac{1}{m_{0}} \simeq k_{e}, \quad \gamma c \simeq X^{0}, \quad \tau \simeq t$.

Utilizing Eqs. (48), (49), (43) and (45), we have

$\frac{\mathrm{d} \gamma}{\mathrm{d} \tau}=\gamma \frac{\mathbf{v}^{\mathrm{t}}}{\mathrm{c}} \frac{\mathbf{F}}{m_{0} c}$

This equation is similar to Eq. (13) upon taking account of the similarities (58), so that we can define $u_{0}$ for special relativistic mechanics:

$u_{0}:=\frac{m_{0} c \mathrm{~d} m}{m} \frac{\mathbf{v}^{\mathrm{t}} \mathbf{F}}{c}$

corresponding to $\dot{q}_{0}$ for plasticity. Since $u_{0}$ is not necessarily restricted to be non-negative here, there exist no switching criteria in the theory of special relativistic mechanics. If $u_{0}=0$, the Newtonian equation $m_{0} \dot{\mathbf{v}}=\mathbf{F}$ is recovered from the special relativistic equation (46) in a way analogous to what we have observed the elastic equation $(9)_{2}$ is recovered from the perfect elastoplastic equation $(9)_{1}$. In addition to the similarity relations (41) and (58) we summarize in Table 2 and Fig. 1 some comparisons between the spacetime of special relativity, special relativistic mechanics and perfect elastoplasticity in the on phase. It is interesting to note that in almost every row of Table 2 the corre- sponding terms are somewhat subtly different in spite of the apparent similarities.

Furthermore, let us consider a massive charged particle with mass $m$ and charge $q$ moving with velocity $\mathbf{v}$ in an (external) electromagnetic field. We neglect the interaction between the field and the charge of the particle. The Lorentz force on the particle is

$\mathbf{F}=q \mathbf{E}+\frac{q}{c} \mathbf{v} \times \mathbf{B}$,

where $\mathbf{E}=\left(E^{1}, E^{2}, E^{3}\right)^{\mathrm{t}}$ and $\mathbf{B}=\left(B^{1}, B^{2}, B^{3}\right)^{\mathrm{t}}$ are the electric field and magnetic induction, respectively. A similar procedure yields Eq. (53) again but with the field strength (in mixed components)

$\mathbf{A}:=\frac{q}{m_{0} c}\left[\begin{array}{cccc}0 & B^{3} & -B^{2} & E^{1} \\ -B^{3} & 0 & B^{1} & E^{2} \\ B^{2} & -B^{1} & 0 & E^{3} \\ E^{1} & E^{2} & E^{3} & 0\end{array}\right]$

superseding the A of Eq. (54). We observe that the field strength in contravariant components $\mathbf{g A}$ is skew-symmetric, that the equation obtained is Lorentz-invariant and linear in the augmented state space, and that the $\mathbf{A}$ obtained belongs to the real Lie algebra $\mathrm{so}(3,1)$ of the Lorentz group $\mathrm{SO}_{o}(3,1)$ acting on $\mathbb{M}^{3+1}$, because it is exactly in the form of Eq. (26) and satisfies Eq. (20). Eq. (53) with $\mathbf{A}$ given in the above is known as the Lorentz equation.

\section{Conclusions}

In the framework of Minkowski spacetime the properties of elastoplasticity, comparison model and elasticity were compared. Cross references between the perfect elastoplastic equations, the spacetime of special relativity, and the equations of special relativistic mechanics were also adopted in this paper, with similarities and distinctions among the theories pointed out. The comparisons and cross references were summarized in Tables 1 and 2 and Fig. 1. Amazingly, we found that, on one hand, the evolution from the Newtonian equations to the special relativistic equations in physical 
science and, on the other hand, the evolution from the elastic constitutive equations to the elastoplastic constitutive equations in mechanical science are highly similar in several facets, notably (a) from a linear theory to a non-linear theory, (b) the state space being enlarged from the usual Euclidean space to Minkowski spacetime, (c) from an $n$-space to a cone of $(n+1)$-space, (d) from a non-bounded state space to a bounded state space, (e) from a non-causal relation of states to a causal relation of augmented states.

\section{References}

[1] H.-K. Hong, C.-S. Liu, Internal symmetry in the constitutive model of perfect elastoplasticity, Int. J. Non-Linear Mech. 35 (2000) 447-466.

[2] H.-K. Hong, C.-S. Liu, Lorentz group $\mathrm{SO}_{o}(5,1)$ for perfect elastoplasticity with large deformation and a consistency numerical scheme, Int. J. Non-Linear Mech. 34 (1999) 1113-1130.

[3] J.L. Anderson, Principles of Relativity Physics, Academic Press, New York, 1967.

[4] A. Das, The Special Theory of Relativity, Springer, New York, 1993.

[5] J.D. Jackson, Classical Electrodynamics, 2nd edition, Wiley, New York, 1975.

[6] G.L. Naber, The Geometry of Minkowski Spacetime, Springer, New York, 1992.
[7] S.B. Kuksin, On classical solutions of the Prandtl-Reuss equations of perfect elastoplasticity, Proc. Roy. Soc. Edinb. A 126 (1996) 1297-1308.

[8] H.-K. Hong, C.-S. Liu, Prandtl-Reuss elastoplasticity: on-off switch and superposition formulae, Int. J. Solids Struct. 34 (1997) 4281-4304.

[9] H.-K. Hong, C.-S. Liu, On behavior of perfect elastoplasticity under rectilinear paths, Int. J. Solids Struct. 35 (1998) 3539-3571.

[10] L. Prandtl, 1924 Spannungsverteilung in plastischen kœrpern, Proceedings of the 1st International Congress on Applied Mechanics, Delft, 1924, pp. 43-54.

[11] E. Reuss, Beruecksichtigung der elastischen formaenderungen in der plastizitaetstheorie, Zeits. Angew. Math. Mech. (ZAMM) 10 (1930) 266-274.

[12] J.F. Cornwell, Group Theory in Physics, Vol. 2, Academic Press, London, 1984.

[13] H.-K. Hong, C.-S. Liu, Internal symmetry in bilinear elastoplasticity, Int. J. Non-Linear Mech. 34 (1999) 279-288.

[14] E.C. Zeeman, Causality implies the Lorentz group, J. Math. Phys. 5 (1964) 490-493.

[15] H.-K. Hong, C.-S. Liu, Lorentz group on Minkowski spacetime for construction of the two basic principles of plasticity, Int. J. Non-Linear Mech., in press.

[16] J.K. Hale, Ordinary Differential Equations, Wiley, New York, 1969.

[17] R. Hill, A general theory of uniqueness and stability in elastic-plastic solids, J. Mech. Phys. Solids 6 (1958) 236-249.

[18] M.J. Sewell, Maximum and Minimum Principles, Cambridge University Press, Cambridge, England, 1987.

[19] N.A. Doughty, Lagrangian Interaction: an Introduction to Relativisitic Symmetry in Electrodynamics and Gravitation, Addison-Wesley, Sydney, 1990. 